\title{
AUMENTO DO NUMERO DE BARRA LAMINADA POR BARRA PERDIDA DO LAMINADOR DEMAG GLQ-VSBM*
}

\author{
Izabelle Lannes Salgueiro Ferreira ${ }^{1}$ \\ Jose Carlos da Silva ${ }^{2}$ \\ Leandro Carlos Pinto de almeida ${ }^{3}$ \\ Leonardo Henrique de Oliveira Mathias ${ }^{4}$
} Bruno Lovatini ${ }^{5}$

\section{Resumo}

Este trabalho teve como finalidade aumentar o índice de rendimento metálico no processo de laminação à quente de aços longos no Laminador Demag, visando reduzir as perdas metálicas com maiores impactos no processo, com foco nas barras laminadas por barras perdidas. Mediante ao cenário atual da economia brasileira, o índice do rendimento metálico no processo de laminação representa um dos maiores impacto no custo de transformação do produto acabado. Para isso dividiu se o trabalho em duas fases, com aplicação da metodologia Seis Sigmas, que consiste em atuar nos problemas de forma ordenada, através da matriz de esforço x impacto. Seis sigmas é um mecanismo utilizado em melhorias de processos em geral, como forte característica identifica os parâmetros mais relevantes e influentes na geração de perdas metálicas no processo de laminação, no qual nos norteou a elaborar um plano de ação de forma sólida, ou seja, ações com menor esforço e maior impacto com foco nas causas fundamentais. Com a implementação do plano de ação, houve um aumento de 0,72\% no índice do rendimento metálico para uma produção 178kt no ano 2015 , com crescente melhora na performance das barras laminadas dos produtos do laminador Demag.

Palavras-chave: rendimento metálico; aços longos; laminador Demag; barras laminadas; barras perdidas;seis sigma; perda metálica.

\section{INCREASING THE NUMBER OF IRON BAR ROLLED FOR IRON BAR LOST OF ROLLING DEMAG GLQ-VSBM}

\section{Abstract}

This work was intended to increase the metal yield index in the hot rolling process of long steel in mill Demag, to reduce metal losses with major impacts in the process, with focus on the iron bar rolled / iron bar lost. Upon the current scenario of the Brazilian economy, the index of the metal yield in the rolling process is one of the largest impact on the cost of processing the final product. Based on this analysis, the proposed work is divided into two stages, applying Six Sigma, which consists to act in orderly fashion problems based on matrix stress $X$ impact. Six sigma is a mechanism used in processes in general improvements, as strong feature identifies the most relevant and influential parameters in the generation of metal losses in the lamination process, which has guided us to prepare an orderly plan of action solid that is, actions with lower effort and greater impact on the process with a focus on fudamental causes. With implementation of the action plan, there was an increase of $0.72 \%$ in the metal yield index for a 178kt production in 2015 with growing improvement in performance of iron bar rolled of mill Demag products.

Keywords: metal yield;long steel; mill Demag; laminated bars; lost bars; six sigma; metal losses.

1 Engenharia Metalúrgica UFF, Engenheira de Processo, Gerencia de Laminação a Quente, Votorantim Siderurgia, Barra Mansa, Rio de Janeiro, Brasil.

2 Técnico Eletromecânica ICT, Especialista em Processos, Gerencia de Laminação a Quente, Votorantim Siderurgia, Barra Mansa, Rio de Janeiro, Brasil.

3 Técnico Mecânica, SENAI, Técnico Especialista em Oficina, Votorantim Siderurgia, Barra Mansa, Rio de Janeiro, Brasil.

4 Engenharia Mecânica UERJ, Coordenador de Produção, Gerencia de Laminação a Quente, Votorantim Siderurgia, Barra Mansa, Rio de Janeiro, Brasil.

5 Técnico Eletrônico, Sabec, Técnico Especialista de automação, Gerencia de Laminação a Quente, Votorantim Siderurgia, Barra Mansa, Rio de janeiro, Brasil. 


\section{INTRODUÇÃO}

Nos últimos cinco anos com a retração do mercado interno, baixa demanda no consumo de aços pelas construções civis, aumento no consumo de aço importado da China com menor custo, escândalo do lava jato na Petrobrás e conflitos políticos entre governantes brasileiros, proporcionou as pequenas, média e grande empresas siderúrgica, fatos que ocasionaram inúmeras situações, demissões em massa, redução no quadro de funcionários, instalação de crises setoriais, falência, agravando o crescimento da economia brasileira.

Os grandes empresários siderúrgicos chegaram à conclusão, que para ser manter operante ao cenário de crise, seria necessário fazer algo diferente, mais por menos, ou seja, maior produtividade com menor custo. Para complicar ainda mais a situação das empresas houve aumento no custo de energia e gás natural, sendo estes os principais fatores juntamente com rendimento metálico que impactam diretamente o custo de transformação dos produtos acabado do processo siderúrgico.

Com isso foram criadas várias frentes de trabalho voltado a redução do custo de transformação do processo de laminação à quente dos produtos via leito de resfriamento barra reta da VSBM GLQ Laminador Demag. No qual utiliza-se processo de deformação de barras de vergalhão com "1 veio" tradicional, "2 e 3 veios" Sliter, processo este que consiste na divisão de barras nos três últimos passes do trem acabador, com alta velocidade e maior produtividade.

Visando ser mais competitiva em custo mediante ao cenário de crise, a Votorantim Siderurgia Barra Mansa GLQ Laminador Demag definiu como projeto estratégico o "Aumento no número de barras laminadas por barras perdidas". O Laminador Demag possui em seu mix, maior percentual dos produtos Sliter, ou seja processo de laminação de maior complexidade e melhor qualidade.

O Demag é um laminador a quente, que utiliza-se tarugos de seção $130 \times 130 \mathrm{~mm}$ com comprimento de 6,50 metros, peso de $0,849 \mathrm{~kg}$, com 2 fornos de reaquecimento fabricado pelas empresas Brasimet e Interfor, possui Trem Desbaste trio aberto, duas dobretas, três tesouras de descarte de cabeça e uma tesoura de picotamento de barras, 19 cadeiras de laminação duo não reversível, sistema de tratamento térmico (Thermex) e leito de resfriamento de $53 \mathrm{~m}$.

Este trabalho teve a finalidade reduzir as perdas metálicas, e consequentemente aumentar o índice de rendimento metálico dos produtos da linha de laminação, com foco nos fatores de maiores impactos negativos a estabilidade do processo, visando reduzir o custo produção e aumentar assim a rentabilidade dos produtos.

Com aplicação da metodologia Seis, mecanismo este utilizado em melhorias de processos, possui como ponto forte, a característica de identificar os parâmetros mais relevantes e influentes na geração de perdas metálicas no processo de laminação, impulsionando-nos a elaborar um plano de ação focado nas causas fundamentais de maiores impactos.

Com implantação do plano de ação, resultou-se um aumento no índice do rendimento metálico para o volume produção anual de 2015 , reduzindo as perdas metálicas de todos os produtos da linha do laminador Demag.

\section{MATERIAIS E MÉTODOS}

Em função da baixa demanda do volume de produção de aços longos, crise instalada na economia brasileira gerou a necessidade da empresa ser competitiva em custo. Para isso a VSBM GLQ Laminador Demag adotou o projeto "Aumento do 
número de barras laminadas por barras perdidas" como planejamento estratégico para o ano de 2015, a fim de aumentar o índice do rendimento metálico.

\section{Alvo do projeto}

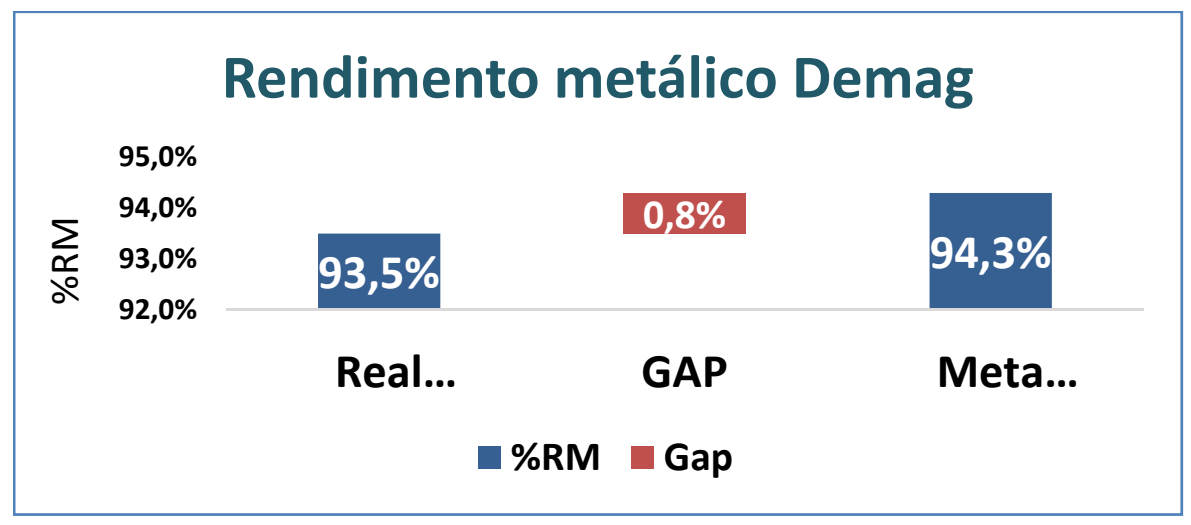

Figura 1. Desafio de meta do RM no 2015 na GLQ Demag

Na fase 1 do projeto, foi realizado histórico dos problemas de maiores impactos no índice do rendimento metálico do ano de 2014 , esta analise direcionou o foco nas perdas metálicas por produtos de maior participação do mix de produção. Com isso foi criado um time multidisciplinar com integrantes do processo, operação, oficina de cilindros, manutenção e automação afim de propor solução para os problemas priorizados.

\section{Histórico do RM por produtos - fase 1}

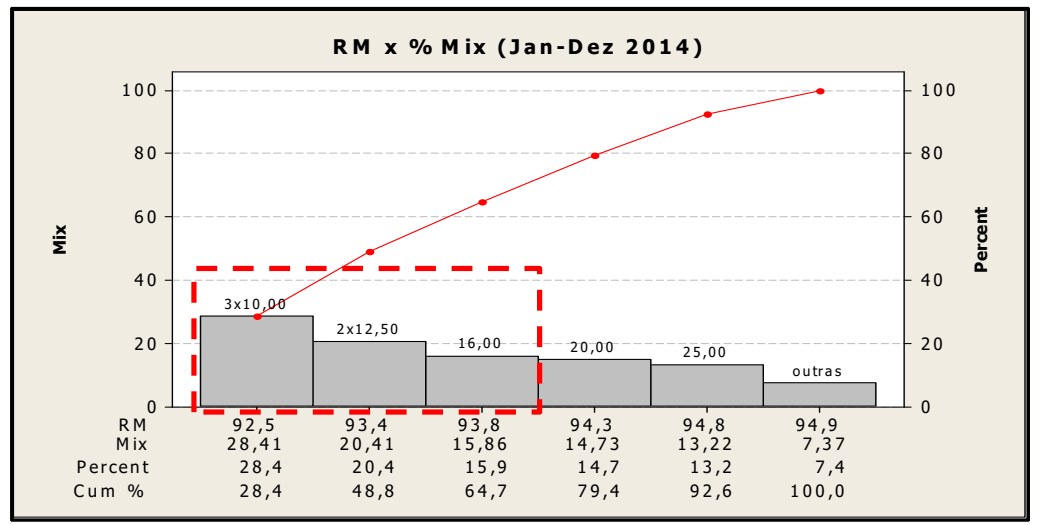

Figura 2. Correlação do RM X MIX dos produtos

Correlação do rendimento metálico com os indicadores dos produtos com maior participação no mix de produção.

\section{Analise de fenômeno - fase 1}



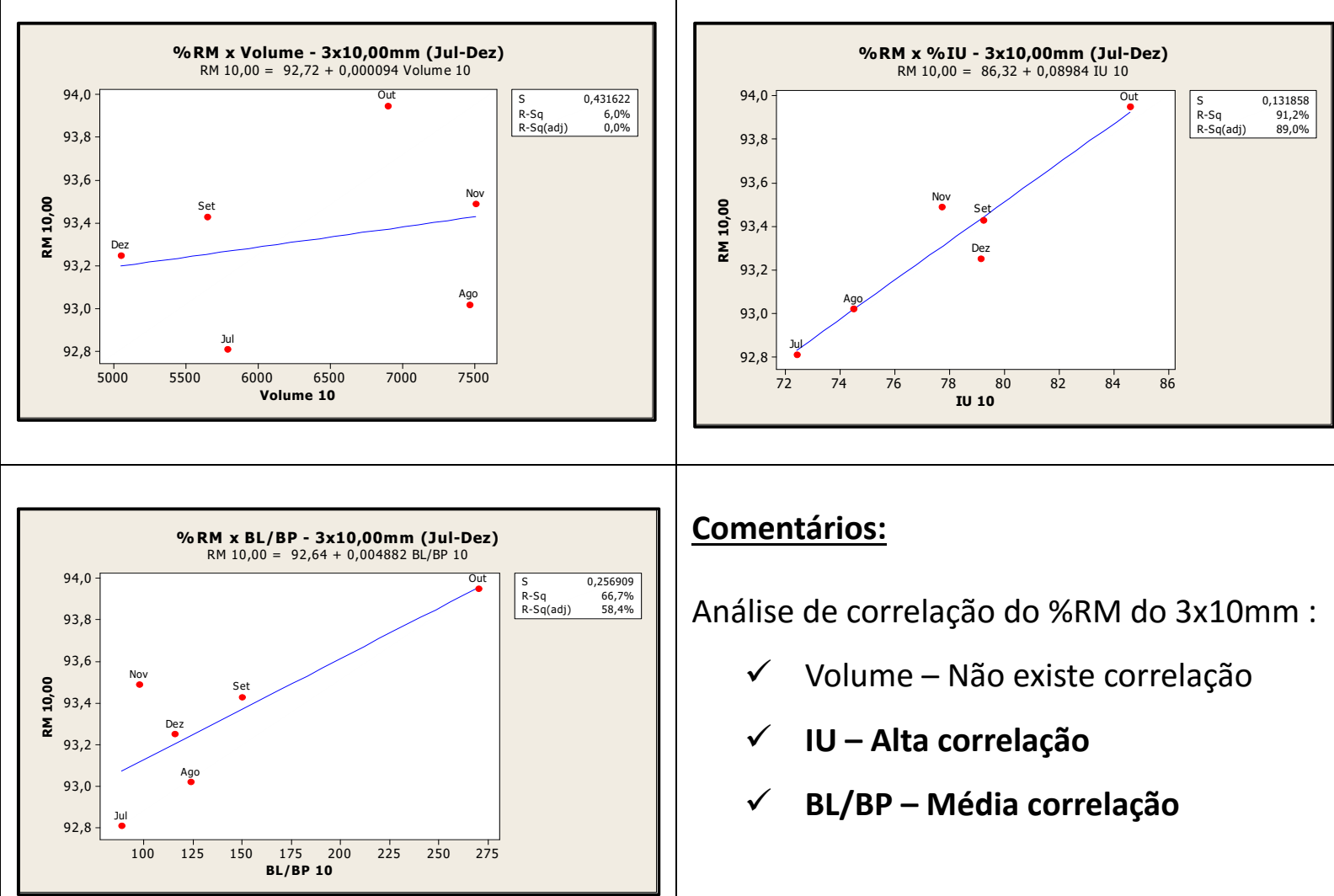

\section{Comentários:}

Análise de correlação do \%RM do $3 \times 10 \mathrm{~mm}$ :

$\checkmark$ Volume - Não existe correlação

$\checkmark$ IU - Alta correlação

$\checkmark$ BL/BP - Média correlação
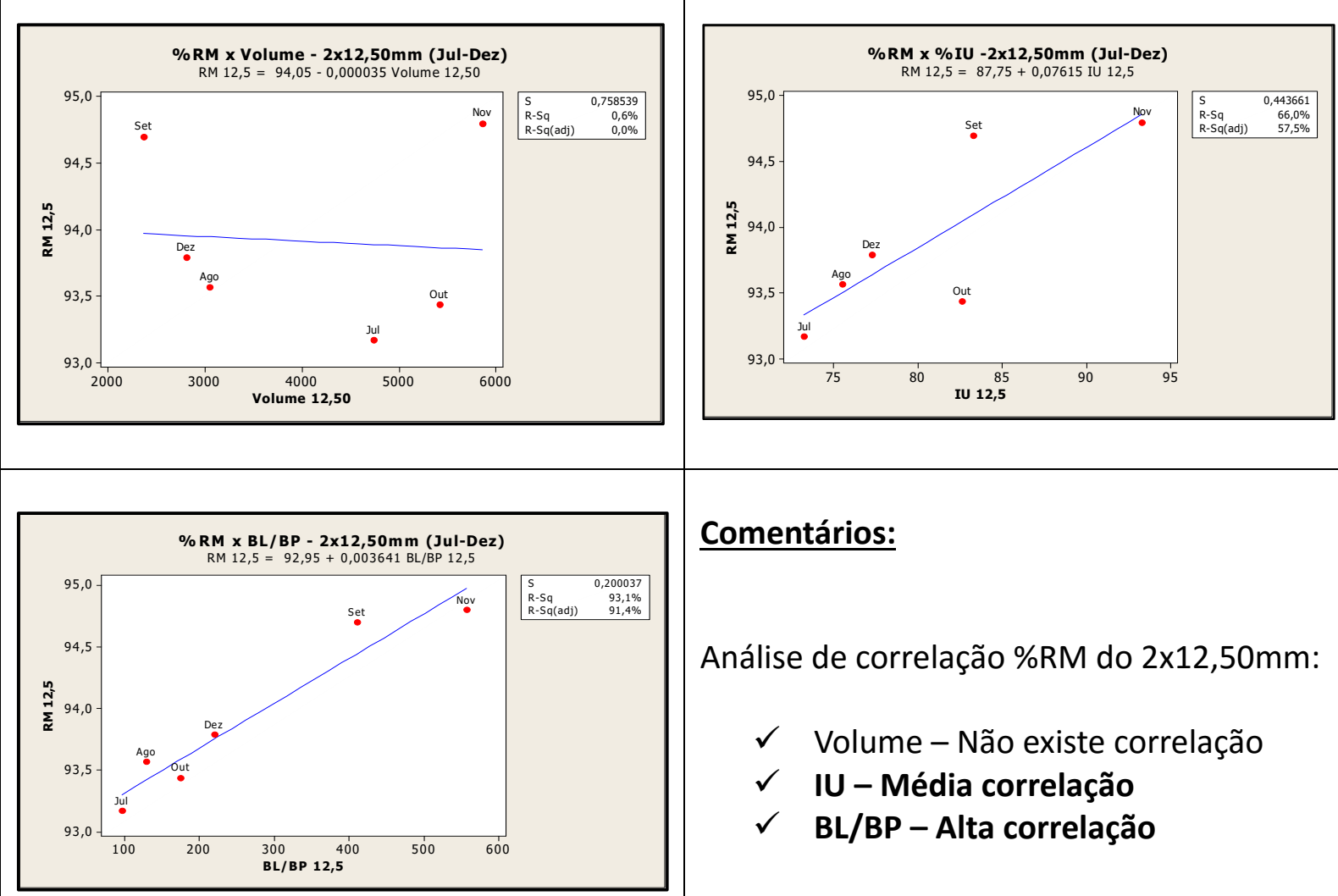

\section{Comentários:}

Análise de correlação \%RM do $2 \times 12,50 \mathrm{~mm}$ :

$$
\begin{array}{ll}
\checkmark & \text { Volume - Não existe correlação } \\
\checkmark & \text { IU - Média correlação } \\
\checkmark & \text { BL/BP - Alta correlação }
\end{array}
$$



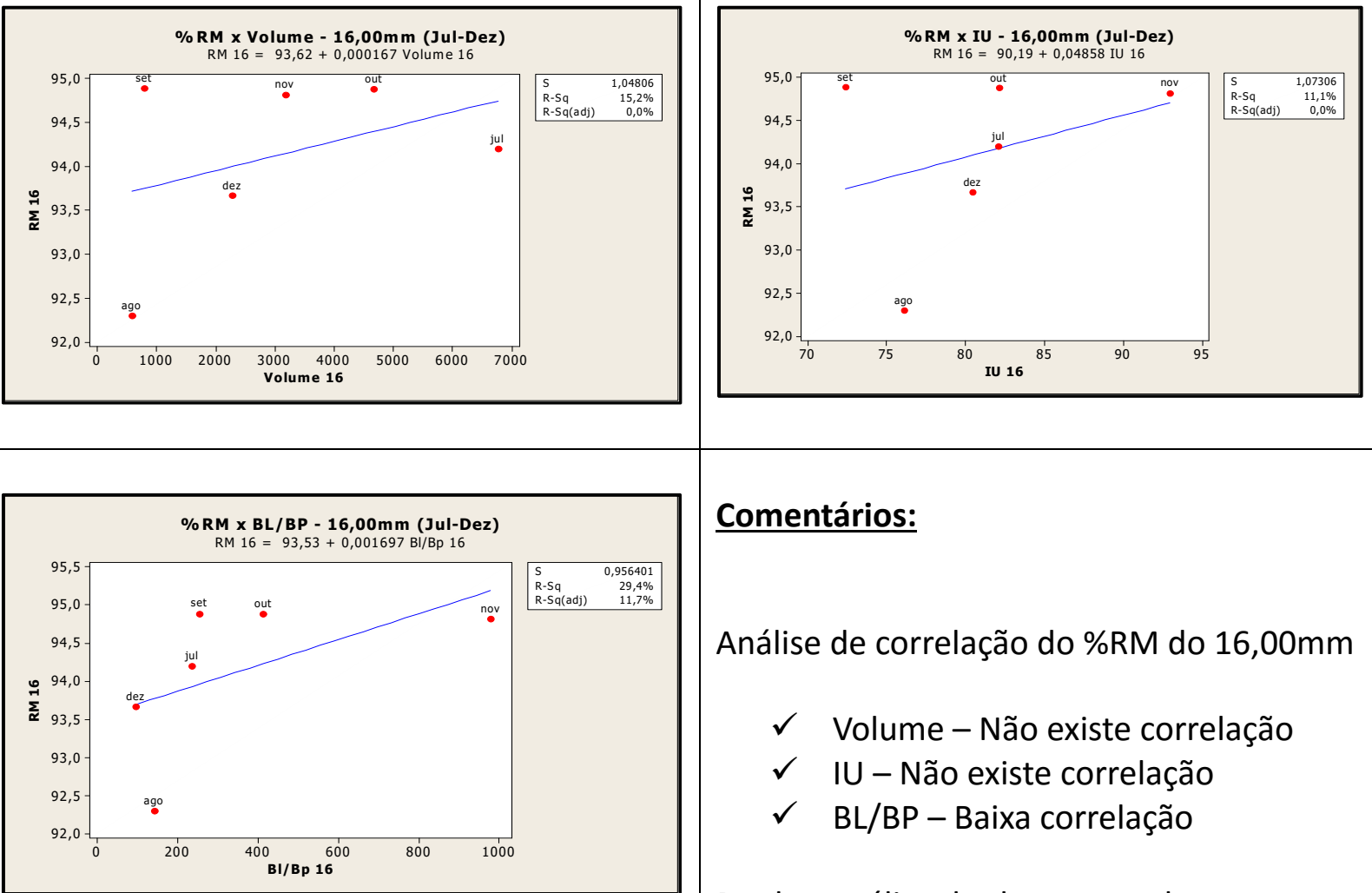

\section{Comentários:}

Análise de correlação do \%RM do 16,00mm

$\checkmark \quad$ Volume - Não existe correlação

$\checkmark \quad$ IU - Não existe correlação

$\checkmark$ BL/BP - Baixa correlação

Perda metálica das barras que batem no final do leito de resfriamento, gerando embolo devido à falha das calhas de frenagem do leito.

A analise de processo norteou as ações do time de projeto, nos equipamentos $x$ responsáveis de maiores impactos nas ocorrências do produto piloto de maior participação no mix de produção anual.

\section{Analise do processo - fase 1}

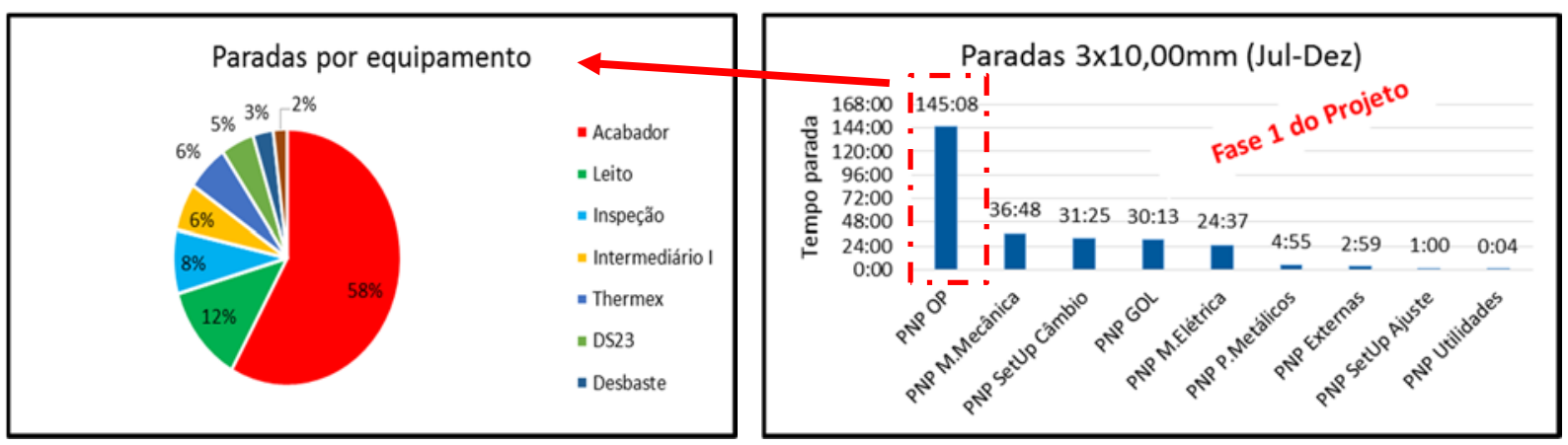

Figura 3 e 4 - Analise de parada do produto CA50 3×10 sliter por equipamento e responsável

Com o levantamento de dados iniciou-se as reuniões de brainstorming do time do projeto para listar possíveis variáveis de processo. 


\section{MATRIZ CAUSA \& EFEITO}

\begin{tabular}{|c|c|c|c|}
\hline \multicolumn{2}{|r|}{ X's do Processo } & \multirow{2}{*}{$\begin{array}{c}\text { TOTAL } \\
10 \\
\end{array}$} & \multirow{2}{*}{$\begin{array}{c}\text { Esforço de Eliminação da } \\
\text { Variável de Entrada }\end{array}$} \\
\hline $\mathrm{X}_{1}$ & Comprimento de tarugo & & \\
\hline$X_{2}$ & Massa linear tarugo & 8 & alto \\
\hline $\mathrm{X}_{3}$ & Perda ao fogo & 5 & baixo \\
\hline $\mathrm{X}_{4}$ & Desponte 870 & 10 & baixo \\
\hline $\mathrm{X}_{5}$ & Desponte 700 & 10 & baixo \\
\hline $\mathrm{X}_{6}$ & Peça de acerto & 8 & baixo \\
\hline $\mathrm{X}_{7}$ & Controle de massa & 8 & baixo \\
\hline $\mathrm{X}_{8}$ & Controle de desponte corte a frio & 5 & baixo \\
\hline $\mathrm{X}_{9}$ & Desalinhamento leito & 8 & baixo \\
\hline $\mathrm{X}_{10}$ & Transferência no leito (pentes) & 8 & alto \\
\hline $\mathrm{X}_{11}$ & Rolos alinhadores & 8 & alto \\
\hline $\mathrm{X}_{12}$ & Variação dos despontes (Automação) & 8 & baixo \\
\hline$X_{13}$ & Otimização dos cortes (Automação) & 8 & baixo \\
\hline $\mathrm{X}_{14}$ & Inspeção laminador & 7 & baixo \\
\hline $\mathrm{X}_{15}$ & Manutenção de bitola & 7 & baixo \\
\hline $\mathrm{X}_{16}$ & Controle temperatura entre os fornos & 6 & baixo \\
\hline $\mathrm{X}_{17}$ & Ajuste de parâmetro de processo & 8 & baixo \\
\hline $\mathrm{X}_{18}$ & Montagem de gaiolas/guias & 8 & baixo \\
\hline$X_{19}$ & $\begin{array}{l}\text { Recebimento de gaiolas/guias no } \\
\text { aminador }\end{array}$ & 8 & baixo \\
\hline $\mathrm{X}_{20}$ & Defeito de tarugo & 8 & baixo \\
\hline
\end{tabular}

Figura 5 - Brainstorming do time do projeto

Com o levantamento das variáveis do processo que impactam diretamente as perdas metálicas, foram priorizadas as mais críticas, numerando-as conforme sua participação, com isso o time de projeto priorização utilizando a matriz de esforço $x$ impacto.
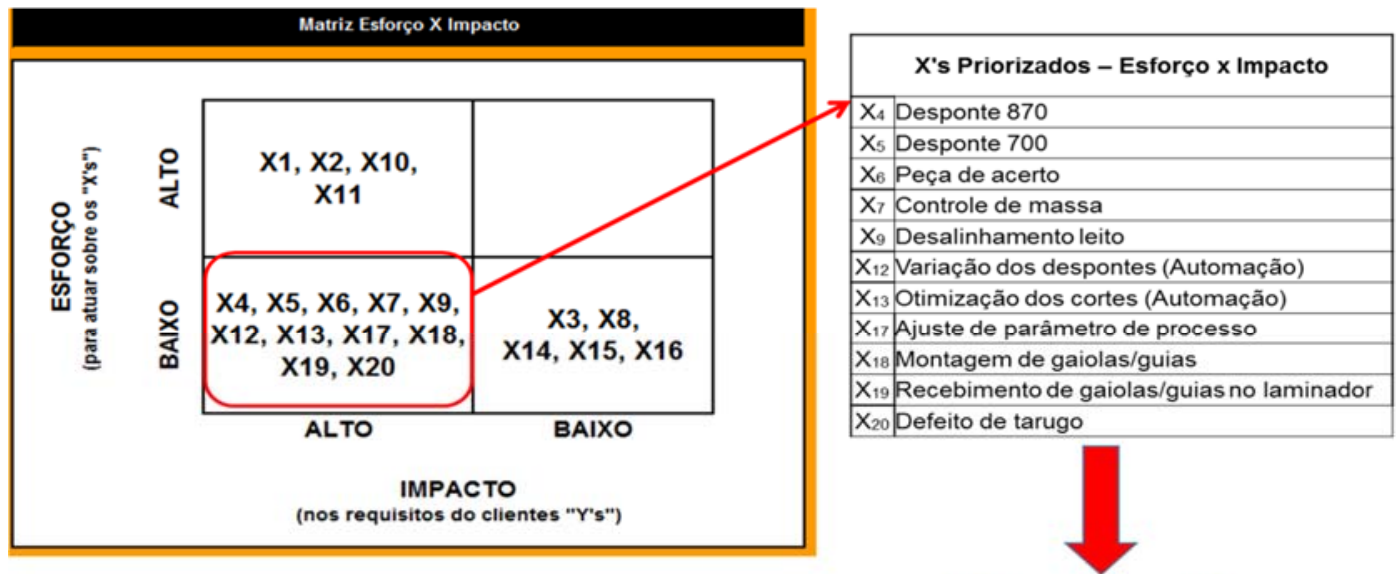

\section{Plano de ação}

Figura 6 - Matriz de esforço x Impacto

Com a priorização das variáveis críticas, foi elaborado o plano de ação com a finalidade de eliminar os fatores que contribuíram negativamente para as perdas metálicas dos produtos, quanto às ocorrências dos equipamentos $\mathrm{x}$ responsáveis. 


\section{Plano de ação - fase 1}

\begin{tabular}{|l|l|l|l|}
\hline \multicolumn{1}{|c|}{ Causa } & \multicolumn{1}{|c|}{ Ação } & Resp. & Status \\
\hline $\begin{array}{l}\text { Utilização de 3 peças para } \\
\text { acerto de bitola (sliter) }\end{array}$ & Realizar procedimento de acerto de bitola na 1ä barra. & José Carlos & OK \\
\hline $\begin{array}{l}\text { Dificuldade na manutenção } \\
\text { da bitola }\end{array}$ & $\begin{array}{l}\text { Revisar procedimento de acerto (PO-071) e receita de processo do } \\
\text { laminador. }\end{array}$ & Implantar check list de partida e pit stop de inspeção (4x turno) & Matheus \\
\hline $\begin{array}{l}\text { Desalinhamento do } \\
\text { material no leito }\end{array}$ & $\begin{array}{l}\text { Alterar o sistema de acionamento dos rolos alinhadores de "seção" para } \\
\text { "individual". }\end{array}$ & OK \\
\hline $\begin{array}{l}\text { Desalinhamento axial de } \\
\text { cilindro }\end{array}$ & $\begin{array}{l}\text { Elaborar procedimento de ajuste do suporte móvel cardan x diâmetro } \\
\text { do cilindro da gaiola. }\end{array}$ & José Carlos & OK \\
\hline $\begin{array}{l}\text { Falha na "mordida"do } \\
\text { material do canal G16 e } \\
\text { G17 }\end{array}$ & $\begin{array}{l}\text { Revisar padrão de montagem de guias (sliter) com utilização do visor } \\
\text { óptico na oficina e gabaritos na área. }\end{array}$ & Leandro & OK \\
\hline
\end{tabular}

Figura 7 - Plano de ação - fase 1

Com a implementação das ações propostas na fase 1 do projeto, reduziu-se $\quad 0,50 \%$ da perda metálica dos produtos da linha de laminação da GLQ Demag

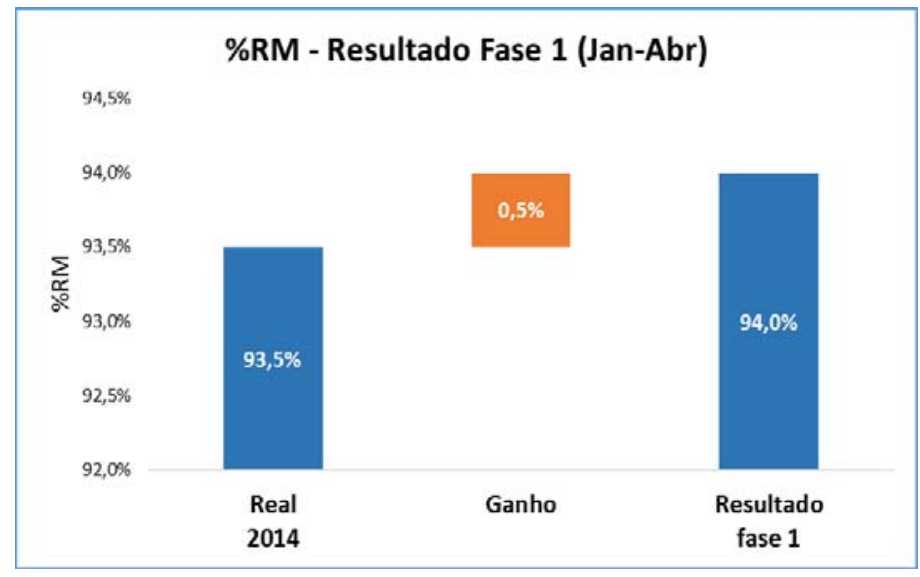

Figura 8 - Resultado no índice do RM - fase 1

$\mathrm{Na}$ fase 2 do projeto com levantamentos de dados e histórico de barras laminadas por barras perdidas por produtos $\mathrm{x}$ equipamentos $\mathrm{x}$ responsáveis, o time de projeto teve foco nas ocorrências que geraram $75 \%$ de impacto nos últimos quatro meses de 2015, definindo que cada responsável por barra perdida no processo de laminação, deveria elaborar plano de ação exclusivamente voltado para tais perdas. Entretanto visando a próxima onda do projeto, que contempla em estabelecer meta de barra perdida por responsável. 


\section{Histórico de sucatas - fase 2}

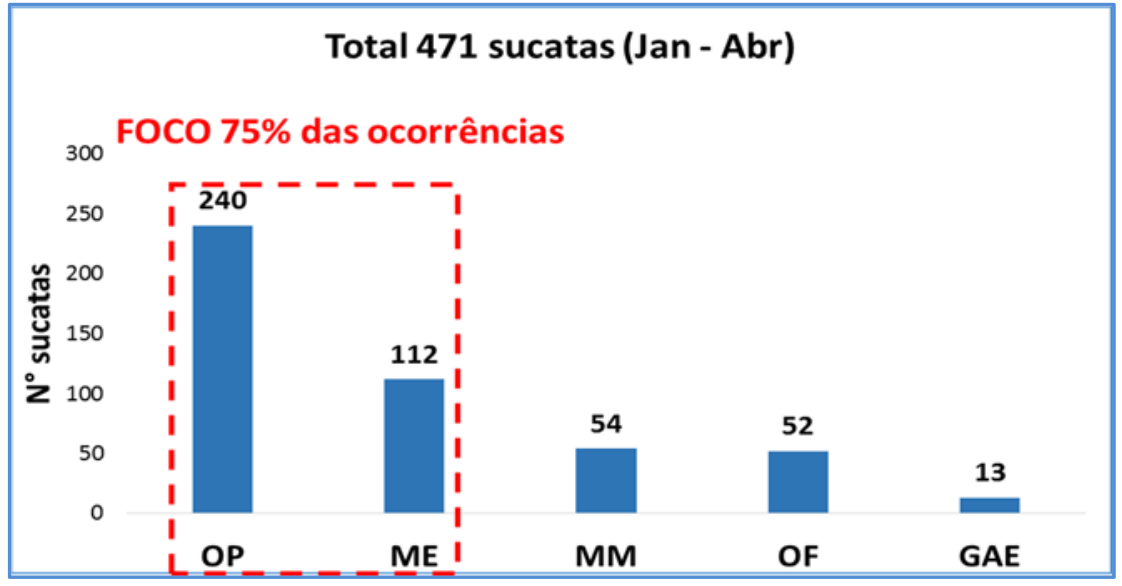

Figura 9 - Sucatas por responsável

\section{Analise de fenômeno - fase 2}

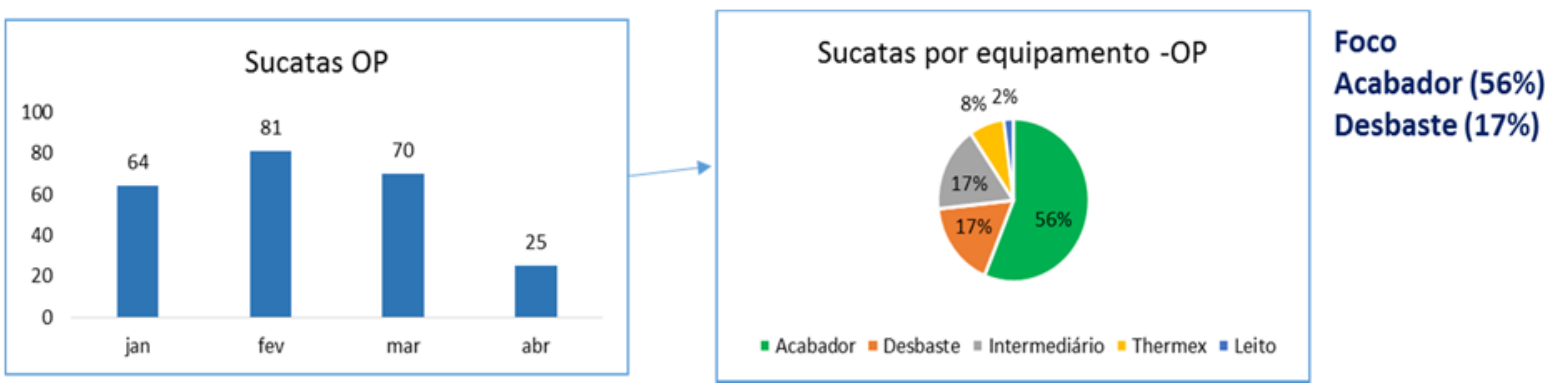

Figura 10 e 11 - Sucatas da operação - 51\% das ocorrências 240 sucatas

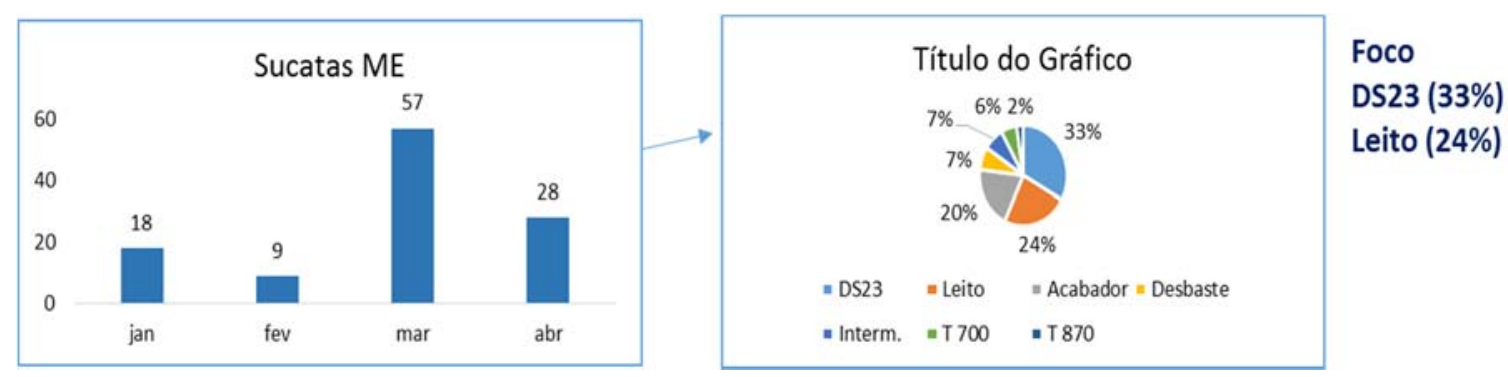

Figura 12 e 13 - Sucatas manutenção elétrica - 24\% das ocorrências - 117 sucatas

\section{Analise do processo - fase 2}

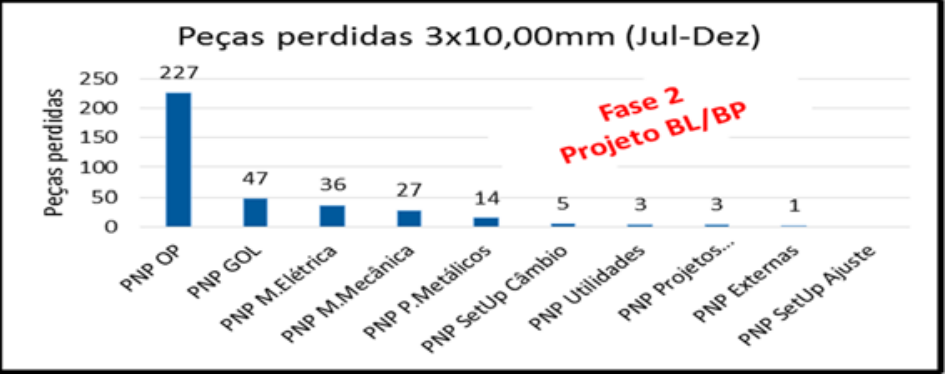

Figura 13 - Barras laminadas / barras perdidas do produto piloto 


\section{Plano de ação - fase 2}

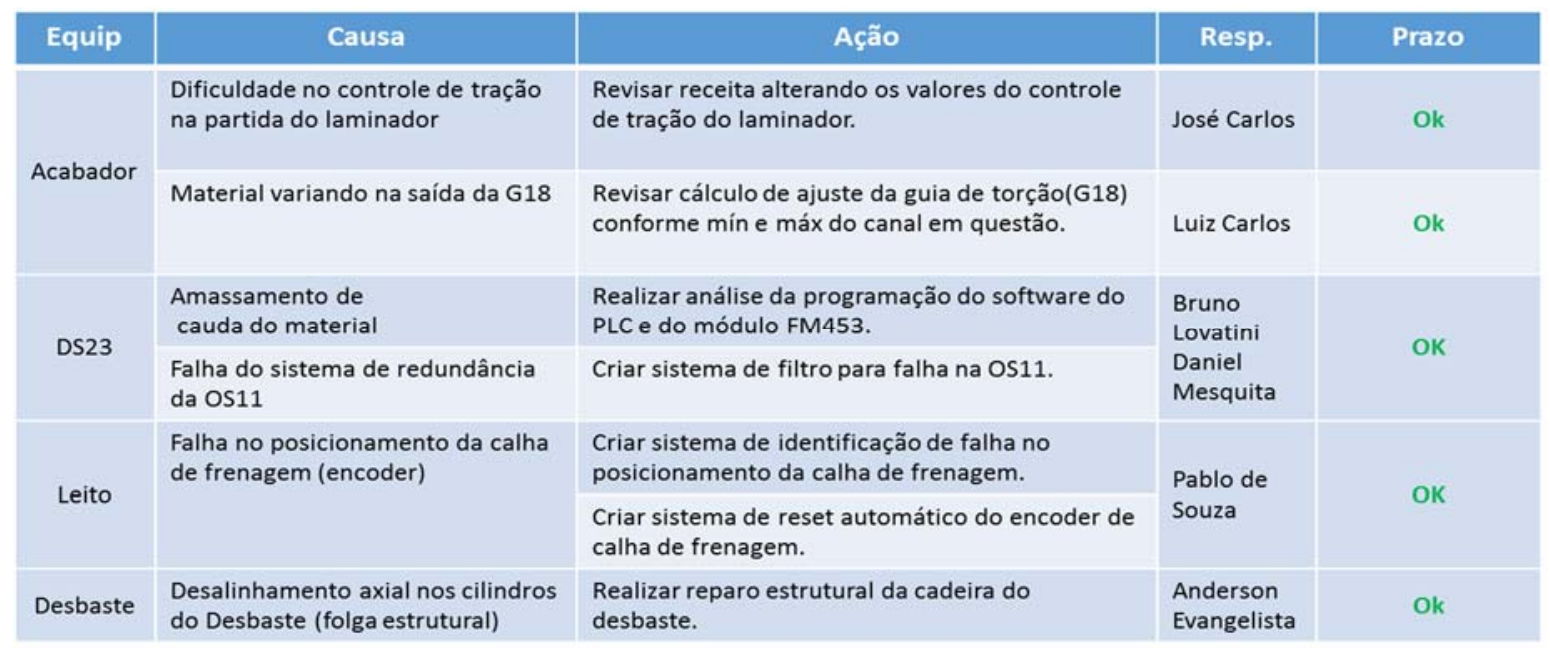

Figura 14 - Plano de ação - fase 1

\section{RESULTADOS E DISCUSSÃO}

Com a proposta de trabalho implementada no projeto BL/BP com foco nos equipamentos $\mathrm{x}$ responsáveis, obteve-se consideravelmente evolução de $74 \%$ no número de barras laminadas por barras perdidas no Laminador GLQ Demag.

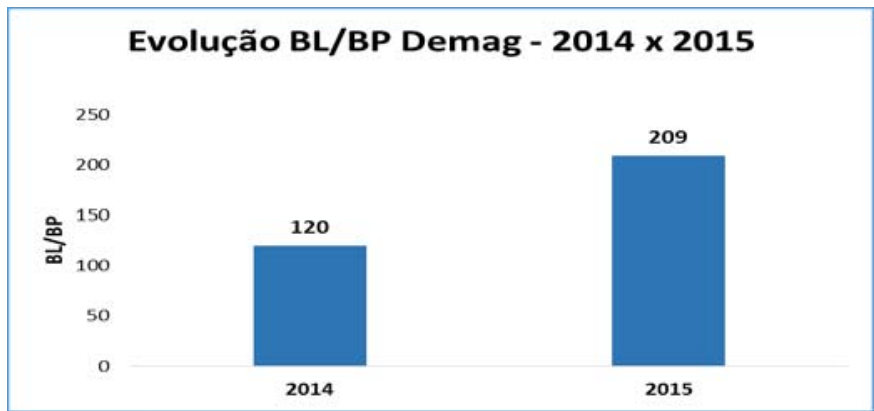

Figura 15 - evolução do BL/BP - fase 2

Após implementação e padronização das ações propostas da FASE 1 + FASE 2 do projeto no laminador Demag, houve considerável aumento de $0,72 \%$ no índice do rendimento metálico do ano de 2015 em relação ao ano de 2014.

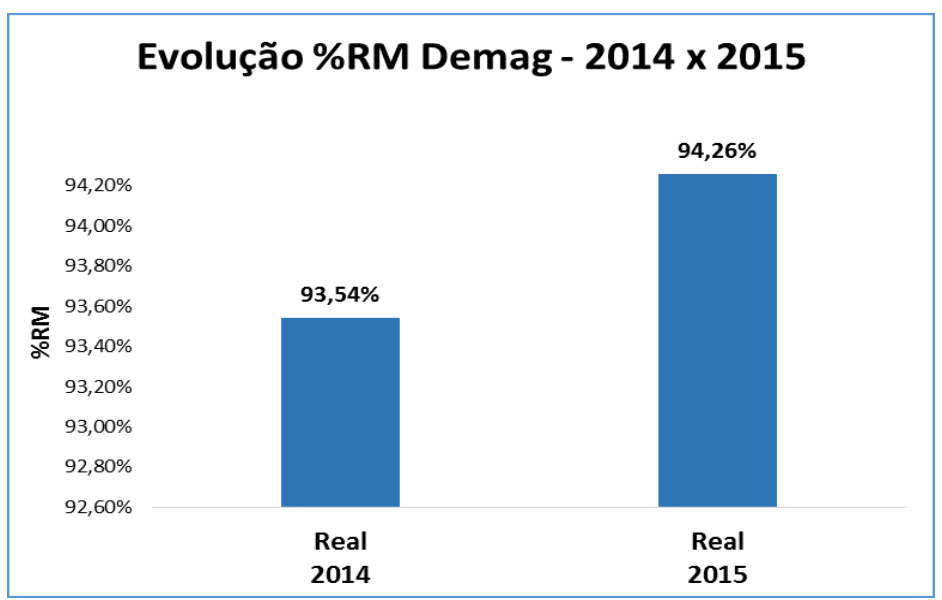

Figura 16 - Aumento no índice de RM - fase 2 
Mesmo com o mix de produção desfavorável, todos os produtos da linha de laminação da GLQ Demag, aumentaram o índice de rendimento metálico.

\begin{tabular}{|c|c|c|}
\hline \multicolumn{3}{|c|}{2014} \\
\hline Bitola & Mix & \%RM \\
\hline $3 \times 10,00$ & $28,0 \%$ & 92,5 \\
\hline $2 \times 12,50$ & $20,0 \%$ & 93,4 \\
\hline 16,00 & $15,8 \%$ & 93,8 \\
\hline 20,00 & $14,7 \%$ & 94,3 \\
\hline
\end{tabular}

\begin{tabular}{|c|c|c|}
\hline \multicolumn{3}{|c|}{2015} \\
\hline Bitola & Mix & \%RM \\
\hline $3 \times 10,00$ & $41,0 \%$ & 93,7 \\
\hline $2 \times 12,50$ & $24,0 \%$ & 94,3 \\
\hline 16,00 & $15,0 \%$ & 94,8 \\
\hline 20,00 & $13,0 \%$ & 94,9 \\
\hline
\end{tabular}

Figura 17 e 18 - Resultados do RM x produtos ano 2014 × 2015

\section{CONCLUSÃO}

A proposta do projeto, em utilizar a metodologia Seis Sigmas com foco no produto CA50 3x10mm que tem maior participação do mix de produção, maior complexidade no processo e menor resultado no índice do rendimento metálico, proporcionou o direcionamento das ações de melhorias para as variáveis críticas de processo, que na maioria dos casos são comuns para todos os produtos da linha de laminação da GLQ Demag. O objetivo deste trabalho foi alcançado e pode-se dizer até superado, pois mesmo havendo aumento em $32 \%$ no mix do produto de maior complexidade em relação ao ano de 2014 , todos os produtos da linha de laminação aumentaram o índice de rendimento metálico em 2015.

É importante destacar que com estes resultados, houve aumento de 0,72\% no índice do rendimento metálico no ano de 2015 comparado ao ano de 2014, gerando faturamento no custo anual da GLQ Demag de R\$ 1.582.227,17. Proporcionando a área GLQ Demag condição de ser competitiva em custo, assegurando a operabilidade dos equipamentos, com condições de assumir novos desafios alinhado ao planejamento estratégico da VSBM e auxiliando o crescimento da VS Brasil.

\section{Agradecimentos}

- Aos supervisores de turno, colaboradores da operação, oficina de cilindros;

- Supervisores da manutenção mecânica; elétrica, automação;

- À gerência de laminação a quente;

- A gerencia de produto e processo.

\section{REFERÊNCIAS}

1 Ernandes Marcos da Silveira Rizzo, Processos de Laminação de Produtos Longos de Aço, ABM, 2010' 